\title{
Associate Higgs and Gauge Boson Production at Hadron Colliders in a Model with Vector Resonances
}

\author{
Alfonso R. Zerwekh * \\ Instituto de Física, Facultad de Ciencias \\ Universidad Austral de Chile \\ Casilla 567, Valdivia, Chile
}

\begin{abstract}
Motivated by new models of dynamical electroweak symmetry breaking that predict a light composite higgs boson, we build an effective lagrangian which describes the Standard Model (with a light Higgs) and vector resonances. We compute the cross section for the associate production of a higgs and a gauge boson. For some values of model parameters we find that the cross section is significantly enhanced with respect to the Standard Model. This enhancement is similar at the LHC and the Tevatron for the same range of resonance mass.
\end{abstract}

\section{Introduction}

One of the main challenges faced today by Particle Physics is to elucidate the nature of electroweak symmetry breaking. This is the only aspect of the Standard Model that still has not been directly tested by experiment. Moreover, there is a general agreement in the sense that, due to the hierarchy and triviality problems, the standard higgs sector is not satisfactory and it really points to the existence of new physics at the $\mathrm{TeV}$ scale.

*alfonsozerwekh@uach.cl 
One possibility for such new physics is the existence of a new strong interaction that dynamically breaks the electroweak symmetry [1. The best known realization of this elegant idea, technicolor, has been challenged by LEP precision measurements. In fact, the original models, which are scaled up versions of QCD, are ruled out. A great progress toward the construction of potentially more successful models was the introduction of walking technicolor which basically means that the interaction is quasi-conformal over a large range of energy. Nevertheless, in general these models predict that the composite scalar (the higgs) in the spectrum must be heavy, while the current experimental data seem to point to the existence of a light higgs.

Very recently a new kind of technicolor models has been proposed [2] whose main characteristic is that technifermions are not in the fundamental representation of the technicolor group. In these models the walking behavior of the coupling constant appears naturally and they are not in conflict with the current limits on the oblique parameters. But the most remarkable feature of these models is that they predict the existence of light composite higgs with a mass around $150 \mathrm{GeV}$.

Inspired by such models, we write down an effective lagrangian which describes the Standard Model with a light higgs and vector resonances which are a general prediction of dynamical symmetry breaking models [3]. The model is minimal in the sense that we assume that any other composite state would be heavier than the vector resonances, and so they are not taken into account, and there are no physical technipions in the spectrum.

In what follows we will study some aspects of such a model. Section 2 is devoted to the construction of the lagrangian. In section 3 we compute the cross section for the associate production of a higgs and a gauge boson and we compare the results with the Standard Model. Finally in section 4 we present our conclusions.

\section{The Lagrangian}

\subsection{Gauge Sector}

We start by noticing that, in general, dynamical electroweak symmetry breaking models predict the existence of composite vector particles ( the so called technirho and techniomega) that mix with the gauge bosons of the Standard Model. In order to describe this mixing, we use a generalization of Vector 
Meson Dominance [4] introduced in [5] and developed in [6]. In this approach we choose a representation where all vector fields transform as gauge fields and they mix through a mass matrix. On the other hand, gauge invariance imposes that the mass matrix has a null determinant. In our case, the lagrangian for the gauge sector can be written as:

$$
\begin{aligned}
\mathcal{L}= & -\frac{1}{4} W_{\mu \nu}^{a} W^{a \mu \nu}-\frac{1}{4} \tilde{\rho}_{\mu \nu}^{a} \tilde{\rho}^{a \mu \nu} \\
& +\frac{M^{2}}{2}\left(\frac{g^{2}}{g_{2}^{2}} W_{\mu}^{a} W^{a \mu}+\tilde{\rho}_{\mu}^{a} \tilde{\rho}^{a \mu}-\frac{2 g}{g_{2}} W_{\mu}^{a} \tilde{\rho}^{a \mu}\right) \\
& -\frac{1}{4} B_{\mu \nu} B^{\mu \nu}-\frac{1}{4} \tilde{\omega}_{\mu \nu} \tilde{\omega}^{\mu \nu} \\
& +\frac{M^{\prime 2}}{2}\left(\frac{g^{\prime 2}}{g_{2}^{\prime 2}} B_{\mu} B^{\mu}+\tilde{\omega}_{\mu} \tilde{\omega}^{\mu}-\frac{2 g^{\prime}}{g_{2}^{\prime}} B_{\mu} \tilde{\omega}^{\mu}\right)
\end{aligned}
$$

where

$$
\begin{aligned}
W_{\mu \nu}^{a} & =\partial_{\mu} W_{\nu}^{a}-\partial_{\nu} W_{\mu}^{a}+g \epsilon^{a b c} W_{\mu}^{b} W_{\nu}^{c} \\
\tilde{\rho}_{\mu \nu}^{a} & =\partial_{\mu} \tilde{\rho}_{\nu}^{a}-\partial_{\nu} \tilde{\rho}_{\mu}^{a}+g_{2} \epsilon^{a b c} \tilde{\rho}_{\mu}^{b} \tilde{\rho}_{\nu}^{c} \\
B_{\mu \nu} & =\partial_{\mu} B_{\nu}-\partial_{\nu} B_{\mu} \\
\tilde{\omega}_{\mu \nu} & =\partial_{\mu} \tilde{\omega}_{\nu}-\partial_{\nu} \tilde{\omega}_{\mu}
\end{aligned}
$$

and $M\left(M^{\prime}\right)$ is the hard mass of the proto-technirho (proto-techniomega). Notice that our lagrangian is written in term of non-physical fields. The physical ones will be obtained by diagonalizing the mass matrix.

By construction, lagrangian (11) is invariant under $S U(2)_{\mathrm{L}} \times U(1)_{\mathrm{Y}}$. The symmetry breaking to $U(1)_{\mathrm{em}}$ will be described by mean of the vacuum expectation value of a scalar field, as in the Standard Model. In other words, we will use an effective gauged linear sigma model as a phenomenological description of the electroweak symmetry breaking.

\section{$2.2 \quad$ Fermions}

As usual, fermions are minimally coupled to gauge bosons through a covariant derivative. Because in our scheme all the vector bosons transform as gauge fields, it is possible to include the proto-technirho and the proto-techniomega 
in an "extended" covariant derivative [6], resulting in the following lagrangian for the fermion sector:

$$
\mathcal{L}=\bar{\psi}_{L} i \gamma^{\mu} D_{\mu} \psi_{L}+\bar{\psi}_{R} i \gamma^{\mu} \tilde{D}_{\mu} \psi_{R}
$$

with

$$
\begin{aligned}
D_{\mu}= & \partial_{\mu}+i \tau^{a} g\left(1-x_{1}\right) W_{\mu}^{a}+i \tau^{a} g_{2} x_{1} \tilde{\rho}_{\mu}^{a} \\
& +i \frac{Y}{2} g^{\prime}\left(1-x_{2}\right) B_{\mu}+i \frac{Y}{2} g_{2}^{\prime} x_{2} \tilde{\omega}_{\mu}
\end{aligned}
$$

and

$$
\tilde{D}_{\mu}=\partial_{\mu}+i \frac{Y}{2} g^{\prime}\left(1-x_{3}\right) B_{\mu}+i \frac{Y}{2} g_{2}^{\prime} x_{3} \tilde{\omega}_{\mu}
$$

The parameters $x_{i}(i=1,2,3)$ play a role similar to fermion delocalization in deconstruction models [7].

A direct coupling between fermions and the vector resonances can appear naturally in technicolor due to extended technicolor interactions. Nevertheless, they must be proportional to the fermion mass and then,they are not important except for the top quark. In what follows, for simplicity, we take $x_{1}=x_{2}=x_{3}=0$.

\subsection{Higgs Sector and Mass Matrices}

In our effective model the higgs sector is assumed to be the same as in the Standard Model except by the possibility of including a direct coupling between the higgs doublet and the vector resonances through an "extended" covariant derivative as was shown for the fermion sector. That is, the lagrangian for the Higgs sector is:

$$
\mathcal{L}=\left(D^{\mu} \Phi\right)^{\dagger}\left(D_{\mu} \Phi\right)-V(\Phi)
$$

where, as usual

$$
V(\Phi)=-\mu^{2} \Phi^{\dagger} \Phi+\lambda\left(\Phi^{\dagger} \Phi\right)^{2}
$$

and

$$
\begin{aligned}
D_{\mu}= & \partial_{\mu}+i \tau^{a} g\left(1-f_{1}\right) W_{\mu}^{a}+i \tau^{a} g_{2} f_{1} \tilde{\rho}_{\mu}^{a} \\
& +i \frac{Y}{2} g^{\prime}\left(1-f_{2}\right) B_{\mu}+i \frac{Y}{2} g_{2}^{\prime} f_{2} \tilde{\omega}_{\mu}
\end{aligned}
$$


This direct coupling may seem natural in a context where the higgs and the vector resonances are both composite states of the same underlying strong sector. Nevertheless, in this work we will avoid it (we choose $f_{1}=f_{2}=0$ ) because, in principle, it can introduce dangerous tree level corrections to the $\rho$ parameter.

Once the electroweak symmetry has been broken, the mass matrix of the vector bosons takes contributions from (II) and from the Higgs mechanism. For the neutral vector bosons, the resulting mass matrix is:

$$
\mathcal{M}_{\text {neutral }}=\frac{v^{2}}{4}\left[\begin{array}{cccc}
(1+\alpha) g^{2} & -\alpha g g_{2} & -g g^{\prime} & 0 \\
-\alpha g g_{2} & \alpha g_{2}^{2} & 0 & 0 \\
-g g^{\prime} & 0 & \left(1+\alpha^{\prime}\right) g^{\prime 2} & -\alpha^{\prime} g^{\prime} g_{2}^{\prime} \\
0 & 0 & -\alpha^{\prime} g^{\prime} g_{2}^{\prime} & \alpha^{\prime} g^{\prime 2}
\end{array}\right]
$$

where

$$
\alpha=\frac{4 M^{2}}{v^{2} g_{2}^{2}}
$$

and

$$
\alpha^{\prime}=\frac{4 M^{\prime 2}}{v^{2} g_{2}^{\prime 2}}
$$

In what follows, in order to simplify our analysis, we will assume that $\alpha^{\prime}=\alpha$ and $g_{2}^{\prime}=g_{2}$. Notice that this assumption implies that the technirho and the techniomega will have the same mass. We will also assume that $M$ is proportional to $g_{2}^{2}$ which is quit natural when we realize that $M$ is a dynamical mass produced by the underlying strong interaction.

On the other hand, the mass matrix for the charged vector bosons can be written as:

$$
\mathcal{M}_{\text {charged }}=\frac{v^{2}}{4}\left[\begin{array}{cc}
(1+\alpha) g^{2} & -\alpha g g_{2} \\
-\alpha g g_{2} & \alpha g_{2}^{2}
\end{array}\right]
$$

We diagonalize the mass matrices in the limit $g_{2} \rightarrow \infty$ and we obtain the following expressions for the physical fields (we have dropped the Lorentz indexes). We write the transformation to physical fields in the limit $g / g_{2} \ll 1$ and we keep terms up to order $g / g_{2}$. 


$$
\begin{aligned}
A & =\frac{g^{\prime}}{\sqrt{g^{2}+g^{\prime 2}}} W^{3}+\frac{g}{\sqrt{g^{2}+g^{\prime 2}}} B+\frac{g g^{\prime}}{g_{2} \sqrt{g^{2}+g^{\prime 2}}} \tilde{\rho}^{3}+\frac{g g^{\prime}}{g_{2} \sqrt{g^{2}+g^{\prime 2}}} \tilde{\omega} \\
Z & =\frac{g}{\sqrt{g^{2}+g^{\prime 2}}} W^{3}-\frac{g^{\prime}}{\sqrt{g^{2}+g^{\prime 2}}} B+\frac{g^{2}}{g_{2} \sqrt{g^{2}+g^{\prime 2}}} \tilde{\rho}^{3}-\frac{g^{\prime 2}}{g_{2} \sqrt{g^{2}+g^{\prime 2}}} \tilde{\omega} \\
\rho^{0} & =-\frac{g}{g_{2}} W^{3}+\tilde{\rho}^{3} \\
\omega & =-\frac{g^{\prime}}{g_{2}} B+\tilde{\omega} \\
W^{ \pm} & =\tilde{W}^{ \pm}+\frac{g}{g_{2}} \tilde{\rho}^{ \pm} \\
\rho^{ \pm} & =\tilde{\rho}^{ \pm}-\frac{g}{g_{2}} \tilde{W}^{ \pm}
\end{aligned}
$$

where

$$
\tilde{W}^{ \pm}=\frac{1}{\sqrt{2}}\left(W^{1} \mp i W^{2}\right)
$$

and

$$
\tilde{\rho}^{ \pm}=\frac{1}{\sqrt{2}}\left(\rho^{1} \mp i \rho^{2}\right)
$$

The relevant Feynman rules, in terms of the physical fields, for the associate production of a higgs and a gauge boson can be found in table 1 while figure 1 shows the Feynman diagrams for the processes studied in this work.

\begin{tabular}{l|l}
\hline Fields in the vertex & Variational derivative of Lagrangian by fields \\
\hline$H \omega_{\mu}^{0} Z_{\nu}$ & $\frac{1}{2} \frac{e^{2} M_{W} \sqrt{\alpha} v}{c_{w}^{3} M_{\rho}} g^{\mu \nu}$ \\
$H \rho_{\mu}^{0} Z_{\nu}$ & $-\frac{1}{2} \frac{e^{2} M_{W} \sqrt{\alpha} v}{c_{w}^{2} M_{\rho} s_{w}} g^{\mu \nu}$ \\
$H \rho_{\mu}^{+} W^{-}{ }_{\nu}$ & $-\frac{1}{2} \frac{e^{2} M_{W} \sqrt{\alpha} v}{M_{\rho} s_{w}^{2}} g^{\mu \nu}$ \\
$\bar{u} d \rho_{\mu}^{+}$ & $\frac{1}{8} \frac{e^{2} \sqrt{2} \sqrt{\alpha} v u d v}{M_{\rho} s_{w}^{2}}\left(1-\gamma^{5}\right) \gamma^{\mu}$ \\
$\bar{u} u \omega_{\mu}^{0}$ & $\frac{1}{24} \frac{e^{2} \sqrt{\alpha} v}{c_{w}^{2} M_{\rho}} \gamma^{\mu}\left(\left(1-\gamma^{5}\right)+4\left(1+\gamma^{5}\right)\right)$ \\
$\bar{u} u \rho_{\mu}^{0}$ & $\frac{1}{8} \frac{e^{2} \sqrt{\alpha} v}{c_{w} M_{\rho} s_{w}}\left(1-\gamma^{5}\right) \gamma^{\mu}$ \\
\hline
\end{tabular}

Table 1: Feynman Rules for the relevant couplings of the vector resonances for the associated production of a higgs and a gauge bosons. The couplings of the $W^{ \pm}$and $Z$ to the quarks are identical, in our limit, to the SM 
We have build our lagrangian based on an extension of vector meson dominance. Another possibility for studying models with vector resonances is to consider the so called hidden local symmetry $[8]$. In this case, the model would correspond to a nonlinear sigma model based on the coset space $U(2)_{L} \otimes U(2)_{R} / U(2)_{V}$. The archetype of this kind of model applied to a strong electroweak model is the so called BESS model 3, 9, (which is based on $\left(S U(2)_{L} \otimes S U(2)_{R}\right) / S U(2)_{V}$ and hence does not have a techniomega). Of course such a model does not include a higgs, nevertheless a "linear" version of the BESS model exists [10] which include scalars in the spectrum.

\section{Results}

We use LanHEP [11] and CompHEP [12] in order to compute the cross section of the associate production of a higgs and a gauge boson at the LHC and the Tevatron (Run II). We choose to work with $\alpha=0.1$ because for values of $\alpha$ of this order, the vector resonances can be light (i.e. $M_{\rho} \approx 250 \mathrm{GeV}$ ) while $g_{2} / g$ is still much bigger than one. As $\alpha$ approaches to one, the vector resonances became too heavy and their observation is increasingly difficult. On the other hand, if $\alpha$ is too small the coupling of the vector resonances to the SM fields are suppressed.

The decay width of the vector resonances were also computed with CompHEP. In the range of masses considered here, the techniomega and the charged technirho decay mainly into fermions while the neutral technirho decays mainly into a pair of $W$ 's.

Notice that $\rho W Z$ and $\rho W A$ vertices do not exist in this model. Indeed, gauge invariance avoid the generation of such couplings from Yang-Mills kinetic terms. For example, a $\rho W A$ vertex coming from Yang-Mills term would violate gauge symmetry in the process $\gamma W \rightarrow \gamma W$. A discussion about this coupling in a similar model can be found in [13.

Our aim in this work is to study the impact of the presence of vector resonances on the higgs production in association with a gauge boson. In particular, we want to compare the predictions of this scenario with the Standard

Model. For this reason we define the following quantity as a measure of the enhancement of the signal over the Standard Model:

$$
\epsilon=\frac{\sigma-\sigma_{\mathrm{SM}}}{\sigma_{\mathrm{SM}}}
$$


where $\sigma$ is the cross section predicted by our effective lagrangian and $\sigma_{\mathrm{SM}}$ is the cross section predicted by the Standard Model.

In figure 2 we show the value of $\epsilon$ as a function of the mass of the technirho $\left(M_{\rho}\right)$ for three values of the higgs mass $\left(M_{H}=115 \mathrm{GeV}\right.$ (solid line), $150 \mathrm{GeV}$ (dashed line) and $200 \mathrm{GeV}$ (dotted line)) for the process $p p \rightarrow H W^{+}$at the LHC. Observe that in this case, the cross section is significatively enhanced with respect to the standard model when the technirho has a mass between $200 \mathrm{GeV}$ and $350 \mathrm{GeV}$. The variation of this enhancement as function of $\alpha$ is shown in figure 4 for $M_{H}=150 \mathrm{GeV}$ and $g 2 / g=10$

On the other hand, when a higgs and a $Z$ are produced (figure 3), the cross section is less enhanced and we expect that this channel will not be sensible to the presence of the vector resonances.

We also compute the cross section of the process $p \bar{p} \rightarrow H W^{+}$at the Tevatron (Run II). The result is shown in figure 5] for $M_{H}=150 \mathrm{GeV}$. Notice that the enhancement in this case is comparable to the prediction for the LHC.

The point in the parameter space we use for studying our model was chosen in order to maximize the deviation from the Standard Model for the selected channel. This procedure allows us to evaluate the possibility of testing the model. Let's now consider the restrictions imposed by data on the electroweak parameters $S, T$ and $U$. This is not the place for performing a detailed calculation of these parameters in our model, nevertheless we expect that the final result must be similar to the one obtained in the minimal BESS model. 3, 9]. Written in the notation of the original authors, this result is:

$$
\begin{aligned}
\epsilon_{1} & =0 \\
\epsilon_{2} & =0 \\
\epsilon_{3} & =-\frac{b}{2}+\left(\frac{g}{g^{\prime \prime}}\right)^{2}
\end{aligned}
$$

where $\epsilon_{1}, \epsilon_{2}, \epsilon_{2}$ are proportional to $T, U$ and $S$ respectively, $g^{\prime \prime}$ is our coupling constant $g_{2}$ and $b$ corresponds to our parameter $x_{1}$ which represents a direct coupling of the proto-technirho to fermions. For the set of parameters used above we obtain $\epsilon_{3}=0.01$ which is disfavored by precision measurements. Nevertheless, we can be consistent with the constrains imposed by precision data by choosing $x_{1}=2\left(g / g_{2}\right)^{2}$. In this case, our results on $\sigma-\sigma_{\mathrm{SM}}$ are modified by a factor 0.60 and an important enhancement remains in the channel $p p \rightarrow H W^{+}$for $M_{\rho}$ around $250 \mathrm{GeV}$. 
At this point a word must be said about the direct search of our vector resonances and the mass limits imposed to them by current data. In general the results of direct search, performed at the Tevatron and LEP, of the technirho and techniomega predicted in usual walking technicolor models applies to our case, except by the fact that we have suposed there are no physical technipions in the spectrum. In this case, considering the technirho decay to charged leptons and a pair of $W$ 's, the technirho (as well as the techniomega) is excluded for $M_{\rho}<206 \mathrm{GeV}[14]$.

\section{Conclusions}

We have constructed an effective lagrangian which represents the Standard Model with a light higgs bosons and vector resonances that mix with the gauge bosons. We fixed the parameter of the model that connects the mass of the new vector bosons with their coupling constant, in such a way that the model were compatible with light resonances.

We want to emphasize that, although this effective lagrangian is inspired by a new technicolor scenario recently proposed in [2], we do not claim that the values chosen for our study represent the low energy limit of the specific models constructed there.

The most obvious process for searching differences between our model and the predictions of the Standard Model is the associate production of a higgs and a gauge boson. We found that the most sensitive channel is the production of the higgs and $W$.

For a range of resonance's mass between $200 \mathrm{GeV}$ and $350 \mathrm{GeV}$ the enhancement of the cross section is significant at both, the LHC and the Tevatron.

\section{Acknowledgements}

The author is grateful to Rogério Rosenfeld for valuable comments

\section{References}

[1] For a recent review see C. T. Hill and E. H. Simmons, Phys. Rept. 381, 235 (2003) [Erratum-ibid. 390, 553 (2004)] arXiv:hep-ph/0203079. 
[2] D. D. Dietrich, F. Sannino and K. Tuominen, Phys. Rev. D 72, 055001 (2005) arXiv:hep-ph/0505059.

[3] For a review of effective models of a strong electroweak symmetry breaking sector with scalar and vector resonances, see D. Dominici, Riv. Nuovo Cim. 20, 1 (1997) arXiv:hep-ph/9711385.

[4] For a modern review on Vector Meson Dominance see, H. B. O'Connell, B. C. Pearce, A. W. Thomas and A. G. Williams, Prog. Part. Nucl. Phys. 39, 201 (1997) arXiv:hep-ph/9501251.

[5] A. R. Zerwekh and R. Rosenfeld, Phys. Lett. B 503, 325 (2001) arXiv:hep-ph/0103159.

[6] A. R. Zerwekh, arXiv:hep-ph/0307130.

[7] R. S. Chivukula, E. H. Simmons, H. J. He, M. Kurachi and M. Tanabashi, Phys. Rev. D 71, 115001 (2005) arXiv:hep-ph/0502162

[8] M. Bando, T. Kugo e K. Yamawaki, Phys. Rep. 164 (1988) 217.

[9] R. Casalbuoni, S. De Curtis, D. Dominici and R. Gatto, Phys. Lett. B155 (1985) 95; Nucl. Phys. B282 (1987) 235;

R. Casalbuoni, P. Chiappetta, A. Deandrea, D. Dominici and R. Gatto, Zeit. für Physik C60 (1993) 315;

R. Casalbuoni, P. Chiappetta, S. De Curtis, F. Feruglio, R. Gatto, B. Mele and J. Terron, Phys. Lett. B249 (1990) 130;

R. Casalbuoni, P. Chiappetta,M.C. Cousinou, S. De Curtis, F. Feruglio, R. Gatto, Phys. Lett. B253 (1991) 275;

L. Antichini,R. Casalbuoni and S. De Curtis,Phys. Lett. B348 (1995) 521.

[10] R. Casalbuoni, S. De Curtis, D. Dominici and M. Grazzini, Phys. Rev. D 56, 5731 (1997) arXiv:hep-ph/9704229.

[11] A. V. Semenov, arXiv:hep-ph/0208011. A. Semenov. LanHEP - a package for automatic generation of Feynman rules. User's manual. INP MSU Preprint 96-24/431, Moscow, 1996; hep-ph/9608488

A. Semenov. Nucl.Inst.\& Meth. A393 (1997) p. 293. 
A. Semenov. LanHEP - a package for automatic generation of Feynman rules from the Lagrangian. Updated version 1.3. INP MSU Preprint 98$2 / 503$.

Home page:http://theory.sinp.msu.ru/ semenov/lanhep.html

[12] E.Boos et al. [CompHEP Collaboration], CompHEP 4.4: Automatic computations from Lagrangians to events, Nucl. Instrum. Meth. A534(2004), p250 hep-ph/0403113.

CompHEP - a package for evaluation of Feynman diagrams and integration over multi-particle phase space. User's manual for version 3.3, hep-ph/9908288

Home page: http://theory.sinp.msu.ru/comphep

[13] R. Rosenfeld, Phys. Rev. D 50, 4283 (1994) arXiv:hep-ph/9403356.

[14] S. Eidelman et al, Phys. Lett. B 592, 1 (2004). 

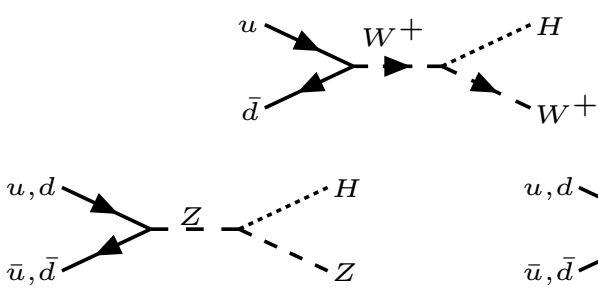

a)

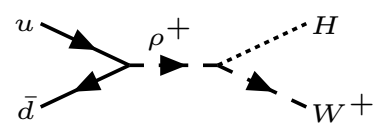

a)

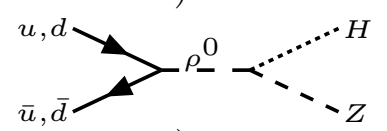

b)

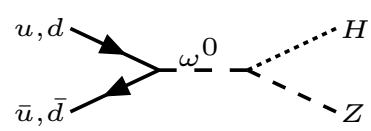

Figure 1: Feynman diagrams for the production of a Higgs and a $W^{+}$(a) , and a Higgs and a $Z$ (b) 


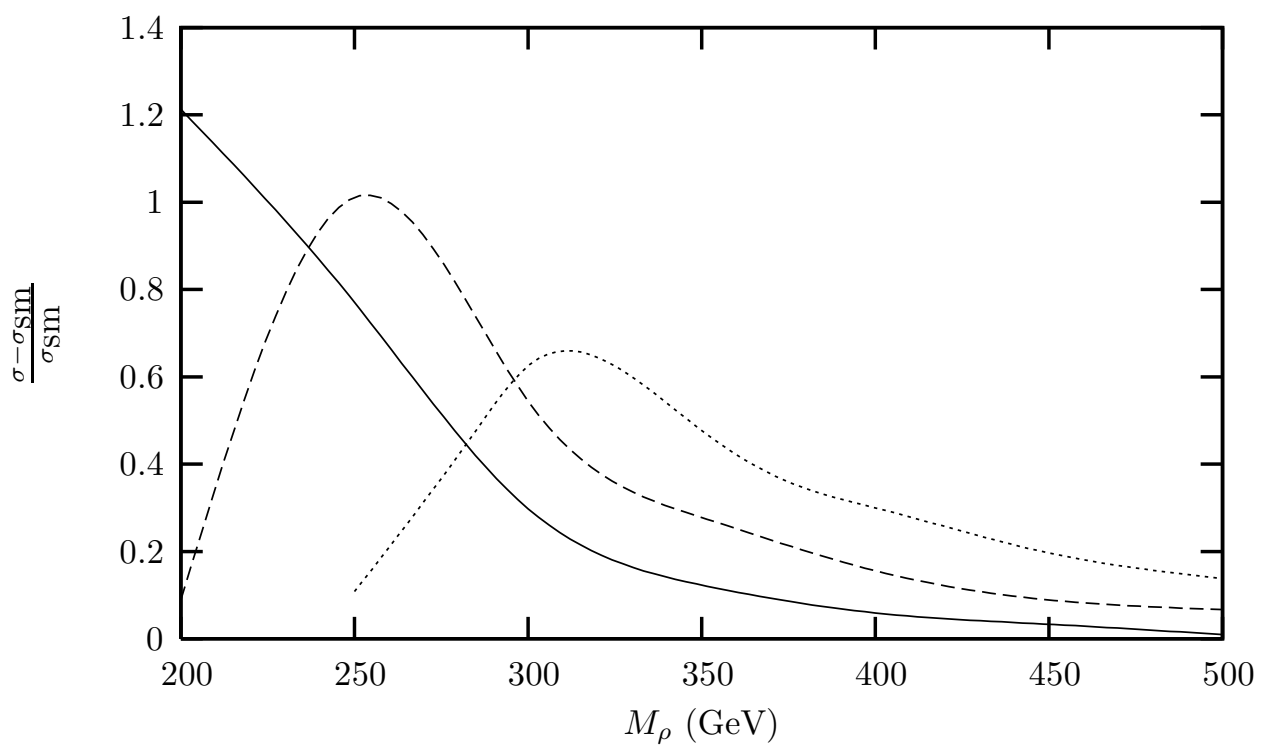

Figure 2: Enhancement of the cross section in the process $p p \rightarrow W^{+} H$ at the LHC for three values of the higgs mass: $M_{H}=115 \mathrm{GeV}$ (solid line), 150 $\mathrm{GeV}$ (dashed line) and $200 \mathrm{GeV}$ (pointed line)

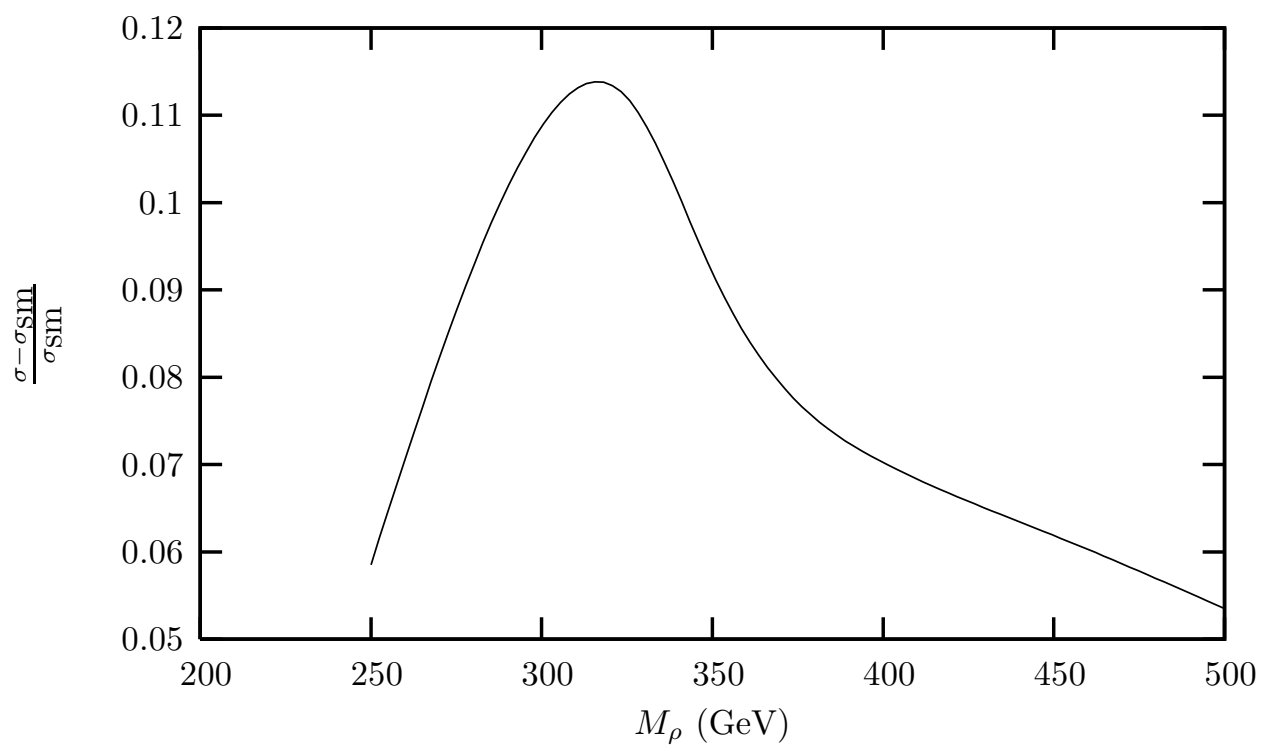

Figure 3: Enhancement of the cross section in the process $p p \rightarrow Z H$ at the LHC for $M_{H}=200 \mathrm{GeV}$ 


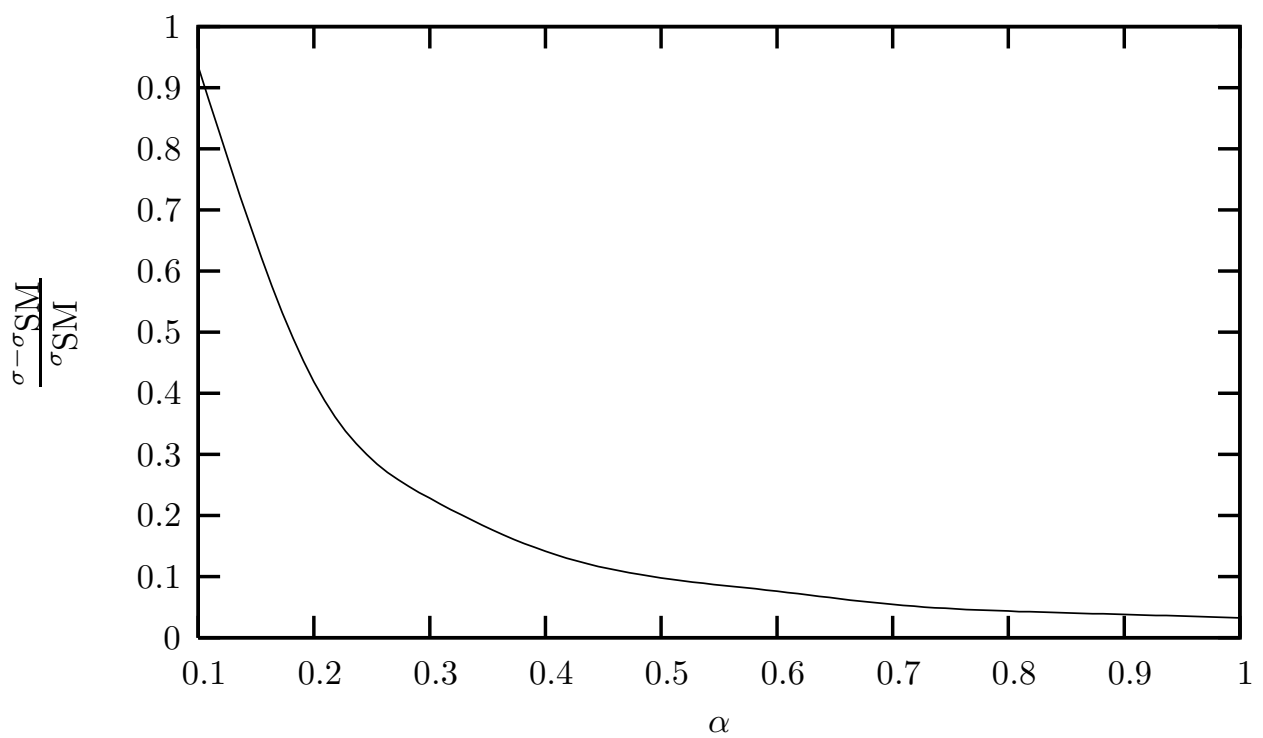

Figure 4: Enhancement of the cross section in the process $p \bar{p} \rightarrow W^{+} H$ at the LHC as a function of $\alpha$ for $M_{H}=150 \mathrm{GeV}$ and $g / g 2=0.1$

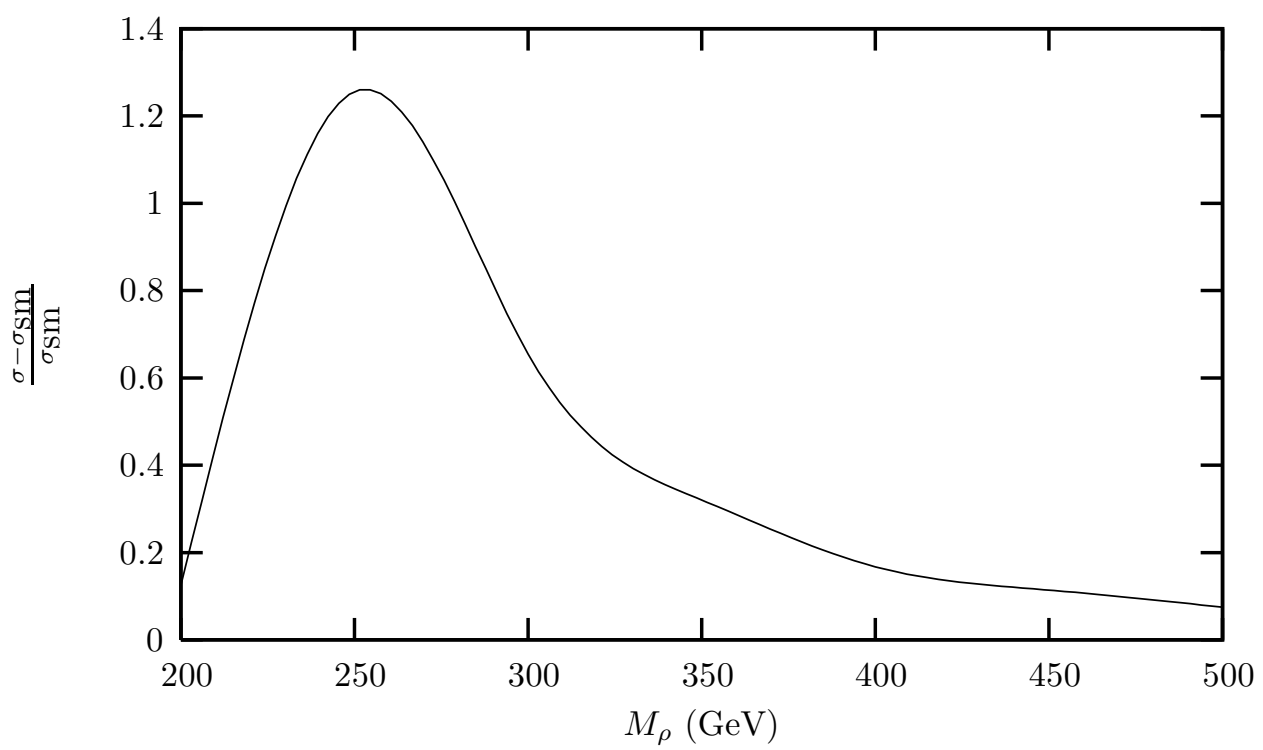

Figure 5: Enhancement of the cross section in the process $p \bar{p} \rightarrow W^{+} H$ at the Tevatron for $M_{H}=150 \mathrm{GeV}$ 